\title{
Correction to: Antipsychotics and dementia in Canada: a retrospective cross-sectional study of four health sectors
}

\author{
Sebastian Rios ${ }^{1}$, Christopher M. Perlman ${ }^{1 *}$, Andrew Costa ${ }^{2,3}$, George Heckman ${ }^{1,2}$, John P. Hirdes ${ }^{1}$ and Lori Mitchell ${ }^{4}$
}

\section{Correction}

Following the publication of this article [1], the authors noticed that the results presented in the results section of the article were erroneously reported in the results section of the abstract. This correction shows both the incorrect and correct values for the results section in the abstract.

The incorrect version is:

The total prevalence of antipsychotic use among older adults with dementia was $26 \%$ in HC, $54 \%$ in ALC, $41 \%$ in CCC, and $48 \%$ in LTC. This prevalence ranged from $38 \%$ (HC) to 73\% (ALC) for those with both behavioral and psychotic symptoms and from $15 \%$ (HC) to $31 \%$ (ALC) among those with no symptoms. The regression models identified a number of variables were related to antipsychotic use in the absence of behavior or psychotic symptoms, such as bipolar disorder $(\mathrm{OR}=6.63$ in CCC; $\mathrm{OR}=5.52$ in LTC), anxious complaints $(\mathrm{OR}=1.54$ in LTC to 2.01 in $C C C)$, and wandering ( $\mathrm{OR}=1.83$ in ALC).

The corrected version is:

The total prevalence of antipsychotic use among older adults with dementia was $19 \%$ in HC, $42 \%$ in ALC, $35 \%$ in CCC, and $37 \%$ in LTC. This prevalence ranged from $39 \%(\mathrm{HC})$ to $\mathbf{7 0 \%}$ (ALC) for those with both behavioral and psychotic symptoms and from $\mathbf{1 2 \%}(\mathrm{HC})$ to $\mathbf{3 2 \%}$ (ALC) among those with no symptoms. The regression models identified a number of variables were related to antipsychotic use in the absence of behavior or psychotic symptoms, such as bipolar disorder $(\mathrm{OR}=\mathbf{5 . 6 3}$ in CCC; $\mathrm{OR}=5.52$ in LTC), anxious complaints $(\mathrm{OR}=1.54$ in LTC to 2.01 in $\mathrm{CCC})$, and wandering ( $\mathrm{OR}=1.83$ in ALC).

\section{Author details}

${ }^{1}$ School of Public Health and Health Systems, University of Waterloo, 200 University Ave W, Waterloo, ON N2L 3G1, Canada. ${ }^{2}$ Schlegel Research
Institute for Aging, Waterloo, ON, Canada. ${ }^{3}$ Department of Clinical Epidemiology and Biostatistics and Medicine, McMaster University, Hamilton, ON, Canada. ${ }^{4}$ Winnipeg Regional Health Authority (WRHA) Home Care Program, Winnipeg, MB, Canada.

Received: 17 May 2018 Accepted: 17 May 2018

Published online: 31 May 2018

Reference

1. Rios, et al. BMC Geriatr. 2017;17:244. https://doi.org/10.1186/s12877-0170636-8.

\footnotetext{
*Correspondence: chris.perlman@uwaterloo.ca

${ }^{1}$ School of Public Health and Health Systems, University of Waterloo, 200

University Ave W, Waterloo, ON N2L 3G1, Canada

Full list of author information is available at the end of the article
}

(c) The Author(s). 2018 Open Access This article is distributed under the terms of the Creative Commons Attribution 4.0 International License (http://creativecommons.org/licenses/by/4.0/), which permits unrestricted use, distribution, and reproduction in any medium, provided you give appropriate credit to the original author(s) and the source, provide a link to the Creative Commons license, and indicate if changes were made. The Creative Commons Public Domain Dedication waiver (http://creativecommons.org/publicdomain/zero/1.0/) applies to the data made available in this article, unless otherwise stated. 